TRANSACTIONS OF THE

AMERICAN MATHEMATICAL SOCIETY

Volume 351, Number 10, Pages 3975-3988

S 0002-9947(99)02265-5

Article electronically published on February 8, 1999

\title{
EXTENDABILITY OF LARGE-SCALE LIPSCHITZ MAPS
}

\author{
URS LANG
}

\begin{abstract}
Let $X, Y$ be metric spaces, $S$ a subset of $X$, and $f: S \rightarrow Y$ a large-scale lipschitz map. It is shown that $f$ possesses a large-scale lipschitz extension $\bar{f}: X \rightarrow Y$ (with possibly larger constants) if $Y$ is a Gromov hyperbolic geodesic space or the cartesian product of finitely many such spaces. No extension exists, in general, if $Y$ is an infinite-dimensional Hilbert space. A necessary and sufficient condition for the extendability of a lipschitz map $f: S \rightarrow Y$ is given in the case when $X$ is separable and $Y$ is a proper, convex geodesic space.
\end{abstract}

\section{INTRODUCTION}

A well-known fact due to E. J. McShane $[\mathrm{M}]$ states that every lipschitz map $f: S \rightarrow \mathbb{R}$ defined on a subset of a metric space $X$ possesses an extension $\bar{f}: X \rightarrow \mathbb{R}$ (i.e. $\bar{f} \mid S=f$ ) satisfying the same lipschitz condition. Another classical result of this type is Kirszbraun's theorem $[\mathrm{K}]$ which applies to lipschitz maps $f: S \rightarrow \mathbb{R}^{n}$, $S \subset \mathbb{R}^{m}$, for $m, n \geq 1$. Recently, V. Schroeder and the author [LS] proved a generalization of Kirszbraun's theorem in the context of metric spaces with curvature bounds in the sense of A. D. Alexandrov. A trimmed, riemannian version of this result reads:

Theorem 1.1 ([LS]). Let $X$ be a complete riemannian manifold with sectional curvature $K_{X} \geq-a^{2}, a \geq 0$, and $Y$ a simply connected, complete riemannian manifold of curvature $K_{Y} \leq-b^{2}, b \geq 0$. Whenever $S \subset X$ and $f: S \rightarrow Y$ is a $\lambda$-lipschitz map with $\lambda b \geq a$, then there exists a $\lambda$-lipschitz extension $\bar{f}: X \rightarrow Y$ of $f$.

As in the classical statement the dimensions of both $X$ and $Y$ are irrelevant (and may as well be infinite). A common feature of these results is that the lipschitz constant of the extension $\bar{f}$ is still the same as for the original map $f$. The aim of the present paper is to enlarge the scope of the above statements - with regard to the geometry of the underlying spaces - at the cost of a weaker property of the extended map. We consider the following large-scale lipschitz condition: a (not necessarily continuous) map $f: X \rightarrow Y$ between two metric spaces $(X, d)$ and $(Y, d)$ is called $(\lambda, \epsilon)$-lipschitz for $\lambda, \epsilon \geq 0$ if

$$
d\left(f(x), f\left(x^{\prime}\right)\right) \leq \lambda d\left(x, x^{\prime}\right)+\epsilon
$$

for all $x, x^{\prime} \in X$. Then $f$ is said to be a $(\lambda, \epsilon)$-quasi-isometric embedding if also $d\left(x, x^{\prime}\right) \leq \lambda d\left(f(x), f\left(x^{\prime}\right)\right)+\epsilon$ for all $x, x^{\prime} \in X$. We generalize McShane's lemma to

Received by the editors August 8, 1997.

1991 Mathematics Subject Classification. Primary 53C20; Secondary 51Kxx, 20F32.

Supported by the Swiss National Science Foundation.

(C)1999 American Mathematical Society 
hyperbolic target spaces in the sense of Gromov [G1] (see section 2 for the definition of $\delta$-hyperbolicity used here).

Theorem 1.2. Let $X$ be a metric space and $Y$ a complete, $\delta$-hyperbolic geodesic space. Then every $(\lambda, \epsilon)$-lipschitz map $f: S \rightarrow Y$ defined on a subset of $X$ possesses $a(\lambda, \epsilon+3 \delta)$-lipschitz extension $\bar{f}: X \rightarrow Y$.

For $Y=\mathbb{R}$ (hence $\delta=0$ ) and $\epsilon=0$, the presented proof reduces to a variant of the simple argument of $[\mathrm{M}]$. For the case $\delta=0$ (i.e. if $Y$ is an $\mathbb{R}$-tree) and $\epsilon=0$, the result was also proved in [LS], as a by-product of the methods developed there. Theorem 1.2 implies that the image of a quasi-isometric embedding $h: Y \rightarrow X$ of a $\delta$-hyperbolic geodesic space $Y$ into a metric space $X$ is a large-scale lipschitz retract in $X$ (compare the question and remark on p. 56 in [G2]; the former is answered affirmatively by Theorem 1.1). In particular, one obtains the following corollary in terms of geometric group theory (see section 2 for detailed comments).

Corollary 1.3. Let $G$ be a finitely generated group and $H \subset G$ a finitely generated subgroup. If $H$ is word hyperbolic, then $H$ admits a lipschitz retraction $\rho: G \rightarrow H$ if and only if $H$ has bounded distortion in $G$.

A simple consequence of McShane's lemma is that every $\lambda$-lipschitz map $f: S \rightarrow$ $\mathbb{R}^{n}$ defined on a subset of a metric space $X$ possesses a $\sqrt{n} \lambda$-lipschitz extension $\bar{f}: X \rightarrow \mathbb{R}^{n}$. Similarly, Theorem 1.2 can be formulated for cartesian products $Y=Y_{1} \times \ldots \times Y_{n}$ of finitely many $\delta$-hyperbolic spaces (cf. Corollary 2.3) and Corollary 1.3 extends to products $H=H_{1} \times \ldots \times H_{n}$ of word hyperbolic groups.

Now let $Y$ be an $n$-dimensional Hadamard manifold, i.e. a simply connected, complete riemannian manifold of curvature $K_{Y} \leq 0$. Let $f: S \rightarrow Y$ be a $(\lambda, \epsilon)$ lipschitz map defined on a subset of a metric space $X$. The above results produce a somewhat inhomogeneous picture: $f$ possesses a $(\sqrt{n} \lambda, \sqrt{n} \epsilon)$-lipschitz extension $\bar{f}: X \rightarrow Y$ if $Y=\mathbb{R}^{n}$, and a $(\lambda, \epsilon+3 \delta)$-lipschitz extension if $Y$ is $\delta$-hyperbolic. One may ask the following:

Question 1.4. In the general case, does there exist a $(\bar{\lambda}, \bar{\epsilon})$-lipschitz extension $\bar{f}: X \rightarrow Y$ of $f$ for some $\bar{\lambda}, \bar{\epsilon}$ depending only on $\lambda, \epsilon$ and $n$ ?

In fact, a scaling argument shows that if this is true, then one may as well take $\bar{\lambda}=\mu \lambda$ for some $\mu$ depending only on $n$. It seems likely that the answer to this question is positive, and that $\mu=\sqrt{n}$ serves the purpose as in the case $Y=\mathbb{R}^{n}$, but we do not know how to prove this. In view of Theorem 1.1 for $a=b=0$ one may even ask if the dependence of $\mu$ on $n$ is necessary at all. This is answered by the following result which is proved in section 3. Given a metric space $\left(Y, d_{Y}\right)$, we call another metric space $\left(X, d_{X}\right)$ an extension of $Y$ if $X \supset Y$ and $d_{X} \mid Y \times Y=d_{Y}$.

Theorem 1.5. For every $n \geq 2$ there exists a metric space extension $X_{n}$ of the euclidean $\mathbb{R}^{n}$ such that there is no $(\lambda, \epsilon)$-lipschitz retraction $\rho: X_{n} \rightarrow \mathbb{R}^{n}$ with $\lambda<n^{1 / 4}$ and $\epsilon \geq 0$. Moreover, there exists an extension $X_{\infty}$ of the separable Hilbert space $\mathbb{R}^{\infty}$ such that there is no large-scale lipschitz retraction $\rho: X_{\infty} \rightarrow \mathbb{R}^{\infty}$ at all.

Here the word "retraction" simply refers to the property $\rho(x)=x$ for $x \in$ $\mathbb{R}^{n}$ and does not include the continuity of $\rho$. (In other words, $\rho: X_{n} \rightarrow \mathbb{R}^{n}$ is an extension of the identity on $\mathbb{R}^{n}$.) The spaces $X_{n}$ are obtained by attaching particular examples of metrized (combinatorial) graphs to $\mathbb{R}^{n}$. Generalizing this 
procedure we formulate a necessary condition for the extendability of a lipschitz map $f: S \rightarrow Y$ in the case when $X \supset S$ is separable and $Y$ is a proper metric space; cf. Proposition 3.2. Moreover, in Theorem 4.4, the obtained condition is also shown to be sufficient provided $Y$ is a locally compact Hadamard space in the sense of Busemann. The discussion extends to large-scale lipschitz maps and yields an equivalent reformulation of Question 1.4 for separable $X$; cf. Theorem 4.5.

\section{Maps into hyperbolic SPACES}

In this section we first recall some definitions, then we prove Theorem 1.2 and discuss the corollaries mentioned in the introduction.

Given a metric space $Y$, a (continuous) curve $\sigma:[a, b] \rightarrow Y$ is called a geodesic if there exists a constant $s \geq 0$ (the speed of $\sigma$ ) such that $d\left(\sigma(t), \sigma\left(t^{\prime}\right)\right)=s\left|t-t^{\prime}\right|$ for all $t, t^{\prime} \in[a, b]$. Then $L(\sigma)=d(x, y)$, where $L$ denotes arc length and $x:=\sigma(a)$, $y:=\sigma(b)$. Conversely, every continuous curve $\sigma^{\prime}:\left[a^{\prime}, b^{\prime}\right] \rightarrow Y$ of minimal length $L\left(\sigma^{\prime}\right)=d(x, y)$ connecting $x$ and $y$ can be reparameterized so that it becomes a geodesic. A metric space $Y$ is called a geodesic space if every pair of points $x, y \in$ $Y$ can be connected by some geodesic. For instance, every complete riemannian manifold is a geodesic space. The image of a geodesic from $x$ to $y$ is denoted $[x, y]$ (which is ambiguous as there may exist different such segments).

Gromov's seminal work [G1] contains several definitions of hyperbolicity for metric spaces, and more have emerged since; see e.g. [B]. However, for geodesic spaces all of them are equivalent up to adjustment of the data describing the hyperbolicity. The most convenient definition for our purposes follows [G1, 6.3]. We abbreviate

$$
\left(y \cdot y^{\prime}\right)_{y_{0}}:=\left[d\left(y, y_{0}\right)+d\left(y^{\prime}, y_{0}\right)-d\left(y, y^{\prime}\right)\right] / 2
$$

if $y_{0}, y, y^{\prime}$ are points in a metric space $Y$. Note that $\left(y \cdot y^{\prime}\right)_{y_{0}}+\left(y_{0} \cdot y^{\prime}\right)_{y}=d\left(y, y_{0}\right)$. By a hinge in a geodesic space $Y$ we mean a pair of geodesic segments $\left[y_{0}, y\right],\left[y_{0}, y^{\prime}\right]$ with a common endpoint $y_{0}$.

Definition 2.1. Let $\delta \geq 0$. A geodesic space $Y$ is called $\delta$-hyperbolic if the following holds: whenever $\left[y_{0}, y\right],\left[y_{0}, y^{\prime}\right]$ is a hinge in $Y$, and $p \in\left[y_{0}, y\right], p^{\prime} \in\left[y_{0}, y^{\prime}\right]$ satisfy $d\left(p, y_{0}\right)=d\left(p^{\prime}, y_{0}\right) \leq\left(y \cdot y^{\prime}\right)_{y_{0}}$, then $d\left(p, p^{\prime}\right) \leq \delta$.

In particular, if $\left[y_{0}, y\right],\left[y_{0}, y^{\prime}\right],\left[y, y^{\prime}\right]$ is a geodesic triangle in a $\delta$-hyperbolic space $Y$, and if $p \in\left[y_{0}, y\right]$, then there exists a point $q \in\left[y_{0}, y^{\prime}\right] \cup\left[y, y^{\prime}\right]$ with $d(q, p) \leq \delta$ (and either $d\left(q, y_{0}\right)=d\left(p, y_{0}\right)$ or $\left.d(q, y)=d(p, y)\right)$. Conversely, it is easily shown that if in a geodesic space $Y$ every geodesic triangle has the property that each of its sides lies within distance $\delta^{\prime}$ of the union of the other two, then $Y$ is $4 \delta^{\prime}$-hyperbolic in the sense of Definition 2.1. For instance, every simply connected, complete riemannian manifold $Y$ with curvature $K_{Y} \leq-b^{2}, b>0$, is $\delta$-hyperbolic for $\delta=$ $b^{-1} \operatorname{arcosh}(3 / 2)$. A 0-hyperbolic geodesic space $Y$ is called an $\mathbb{R}$-tree (see [MoSh] for other characterizations of $\mathbb{R}$-trees).

Now we prove Theorem 1.2. As mentioned in the introduction, the proof extends the simple argument of McShane $[\mathrm{M}]$. The completeness of $Y$ is only used in the case $\delta=0$.

Proof of Theorem 1.2. We fix a reference point $y_{0} \in Y$ and abbreviate $\|y\|:=$ $d\left(y, y_{0}\right)$ and

$$
\left(y \cdot y^{\prime}\right):=\left(y \cdot y^{\prime}\right)_{y_{0}}=\left[\|y\|+\left\|y^{\prime}\right\|-d\left(y, y^{\prime}\right)\right] / 2
$$


for $y, y^{\prime} \in Y$. For $x \in X$ and $z \in S$ we define

$$
\mu(x, z):=\max \{0,\|f(z)\|-\lambda d(x, z)-\epsilon / 2\}
$$

and $\bar{\mu}(x):=\sup _{z \in S} \mu(x, z)$. Note that $\bar{\mu}(x)<\infty$ since

$$
\begin{aligned}
\mu(x, z) & \leq \max \{0,\|f(y)\|+d(f(y), f(z))-\lambda d(x, z)-\epsilon / 2\} \\
& \leq \max \{0,\|f(y)\|+\lambda d(y, z)+\epsilon-\lambda d(x, z)-\epsilon / 2\} \\
& \leq\|f(y)\|+\lambda d(x, y)+\epsilon / 2
\end{aligned}
$$

for all $y, z \in S$. Moreover, the triangle inequality yields $\bar{\mu}(x)+\lambda d\left(x, x^{\prime}\right) \geq \bar{\mu}\left(x^{\prime}\right)$ whenever $x, x^{\prime} \in X$, thus $\bar{\mu}: X \rightarrow \mathbb{R}$ is $\lambda$-lipschitz. For every $z \in S$ we choose a geodesic segment $s(z)=\left[y_{0}, f(z)\right] \subset Y$, and for every pair $(x, z) \in X \times S$ we let $p(x, z) \in s(z)$ denote the point with $\|p(x, z)\|=\mu(x, z)$. By the definition of $\mu$ we have $d(p(x, z), f(z))=\lambda d(x, z)+\epsilon / 2$ unless $p(x, z)=y_{0}$. We claim that

$$
d\left(p(x, z), p\left(x^{\prime}, z^{\prime}\right)\right) \leq \max \left\{\left|\mu(x, z)-\mu\left(x^{\prime}, z^{\prime}\right)\right|+\delta, \lambda d\left(x, x^{\prime}\right)+2 \delta\right\}
$$

whenever $x, x^{\prime} \in X$ and $z, z^{\prime} \in S$. If $\mu(x, z) \leq\left(f(z) \cdot f\left(z^{\prime}\right)\right)$ or $\mu\left(x^{\prime}, z^{\prime}\right) \leq(f(z)$. $f\left(z^{\prime}\right)$ ), then clearly

$$
d\left(p(x, z), p\left(x^{\prime}, z^{\prime}\right)\right) \leq\left|\mu(x, z)-\mu\left(x^{\prime}, z^{\prime}\right)\right|+\delta .
$$

In the remaining case we choose a segment $\left[f(z), f\left(z^{\prime}\right)\right]$ and let $q, q^{\prime} \in\left[f(z), f\left(z^{\prime}\right)\right]$ be the points with $d(q, f(z))=d(p(x, z), f(z))$ and $d\left(q^{\prime}, f\left(z^{\prime}\right)\right)=d\left(p\left(x^{\prime}, z^{\prime}\right), f\left(z^{\prime}\right)\right)$. Then $d\left(p(x, z), p\left(x^{\prime}, z^{\prime}\right)\right) \leq d\left(q, q^{\prime}\right)+2 \delta$, and

$$
\begin{aligned}
d\left(q, q^{\prime}\right) & =d\left(f(z), f\left(z^{\prime}\right)\right)-d(p(x, z), f(z))-d\left(p\left(x^{\prime}, z^{\prime}\right), f\left(z^{\prime}\right)\right) \\
& \leq \lambda d\left(z, z^{\prime}\right)+\epsilon-\lambda d(x, z)-\epsilon / 2-\lambda d\left(x^{\prime}, z^{\prime}\right)-\epsilon / 2 \\
& \leq \lambda d\left(x, x^{\prime}\right),
\end{aligned}
$$

which proves the claim. Next we construct a $(\lambda, 2 \delta)$-lipschitz map $\bar{p}: X \rightarrow Y$ satisfying $d(\bar{p}(y), f(y)) \leq \epsilon / 2+\delta$ for $y \in S$. Consider first the case $\delta=0$. For a given $x \in X$ we choose a sequence $z_{1}, z_{2}, \ldots$ in $S$ such that $\lim _{i \rightarrow \infty} \mu\left(x, z_{i}\right)=$ $\bar{\mu}(x)$. It follows from (1) that the points $p\left(x, z_{i}\right)$ form a Cauchy sequence. By the completeness of $Y$ this sequence possesses a limit $\bar{p}(x) \in Y$ which does not depend on the choice of the points $z_{i}$. Using (1) again we see that the map $\bar{p}$ obtained this way is $\lambda$-lipschitz since $\bar{\mu}$ is. Let $y \in S$. If $\bar{p}(y) \in s(y)$, then

$$
d(\bar{p}(y), f(y))=\|f(y)\|-\bar{\mu}(y) \leq\|f(y)\|-\mu(y, y) \leq \epsilon / 2 .
$$

If $\bar{p}(y) \notin s(y)$, then we pick $z_{1}, z_{2}, \ldots$ in $S$ such that $p\left(y, z_{i}\right) \rightarrow \bar{p}(y)$ as $i \rightarrow \infty$ and $p\left(y, z_{i}\right) \notin s(y)$ for each $i$. Since $Y$ is an $\mathbb{R}$-tree we get

$$
\begin{aligned}
d\left(p\left(y, z_{i}\right), f(y)\right) & =d\left(f(y), f\left(z_{i}\right)\right)-d\left(p\left(y, z_{i}\right), f\left(z_{i}\right)\right) \\
& \leq \lambda d\left(y, z_{i}\right)+\epsilon-\lambda d\left(y, z_{i}\right)-\epsilon / 2 \\
& =\epsilon / 2
\end{aligned}
$$

hence $d(\bar{p}(y), f(y)) \leq \epsilon / 2$. Now consider the case $\delta>0$. For every $x \in X$ we choose a point $z_{x} \in S$ such that $\mu\left(x, z_{x}\right) \geq \bar{\mu}(x)-\delta$, and for $y \in S$ we assume additionally that $\mu\left(y, z_{y}\right) \geq \mu(y, y)$. Then we put $\bar{p}(x):=p\left(x, z_{x}\right)$. For $x, x^{\prime} \in X$ we have

$$
\left|\mu\left(x, z_{x}\right)-\mu\left(x^{\prime}, z_{x^{\prime}}\right)\right| \leq\left|\bar{\mu}(x)-\bar{\mu}\left(x^{\prime}\right)\right|+\delta \leq \lambda d\left(x, x^{\prime}\right)+\delta,
$$


thus $\bar{p}$ is $(\lambda, 2 \delta)$-lipschitz due to (1). Let $y \in S$. If $\|\bar{p}(y)\|=\mu\left(y, z_{y}\right) \leq\left(f(y) \cdot f\left(z_{y}\right)\right)$, then clearly

$$
\begin{aligned}
d(\bar{p}(y), f(y)) & \leq\|f(y)\|-\mu\left(y, z_{y}\right)+\delta \\
& \leq\|f(y)\|-\mu(y, y)+\delta \\
& \leq \epsilon / 2+\delta .
\end{aligned}
$$

Similarly, if $\|\bar{p}(y)\|>\left(f(y) \cdot f\left(z_{y}\right)\right)$, then

$$
\begin{aligned}
d(\bar{p}(y), f(y)) & \leq d\left(f(y), f\left(z_{y}\right)\right)-d\left(p\left(y, z_{y}\right), f\left(z_{y}\right)\right)+\delta \\
& \leq \lambda d\left(y, z_{y}\right)+\epsilon-\lambda d\left(y, z_{y}\right)-\epsilon / 2+\delta \\
& =\epsilon / 2+\delta .
\end{aligned}
$$

Hence, in both cases, $\bar{p}$ has the desired properties. Finally, we define $\bar{f}: X \rightarrow Y$ such that $\bar{f}=\bar{p}$ on $X \backslash S$ and $\bar{f}=f$ on $S$; we know that both $\bar{f} \mid X \backslash S$ and $\bar{f} \mid S$ are $(\lambda, \epsilon+2 \delta)$-lipschitz. Let $x \in X \backslash S$ and $y \in S$. Then

$$
\begin{aligned}
d(\bar{f}(x), \bar{f}(y)) & \leq d(\bar{p}(x), \bar{p}(y))+d(\bar{p}(y), f(y)) \\
& \leq \lambda d(x, y)+2 \delta+\epsilon / 2+\delta,
\end{aligned}
$$

thus $\bar{f}$ is a $(\lambda, \epsilon+3 \delta)$-lipschitz extension of $f$.

We note that the above argument contains a proof of the following approximation result which is clearly optimal.

Lemma 2.2. Let $X$ be a metric space, $Y$ a complete $\mathbb{R}$-tree, and $f: X \rightarrow Y$ a $(\lambda, \epsilon)$-lipschitz map. Then there exists a $\lambda$-lipschitz map $f^{\prime}: X \rightarrow Y$ with

$$
d\left(f^{\prime}(x), f(x)\right) \leq \epsilon / 2
$$

for all $x \in X$.

Next we discuss Corollary 1.3. Let $G$ be a finitely generated group and $A \subset G$ a finite generating system with $A=A^{-1}$, i.e. $a \in A$ if and only if $a^{-1} \in A$. For $g \in G$ one denotes by $\|g\|_{A}$ the length of a shortest word over $A$ representing $g$ (where $\|e\|_{A}:=0$ for the neutral element of $G$ ). The word metric on $G$ with respect to $A$ is the left-invariant metric defined by $d_{A}\left(g, g^{\prime}\right):=\left\|g^{-1} g^{\prime}\right\|_{A}$ for $g, g^{\prime} \in G$. Now let $H \subset G$ be a subgroup with a finite generating system $B=B^{-1}$. Clearly there exists a constant $\lambda \geq 1$ such that $\|h\|_{A} \leq \lambda\|h\|_{B}$ for all $h \in H$ (one can take $\lambda=1$ if $B \subset A$ ). On the other hand, $\tau: \mathbb{R} \rightarrow \mathbb{R}$ is called a distortion function for $H$ in $G$ (with respect to $A, B$ ) if

$$
\|h\|_{B} \leq \tau\left(\|h\|_{A}\right)\|h\|_{A}
$$

for all $h \in H$. Then $H$ is said to have bounded distortion in $G$ if, for some generating sets $A \subset G$ and $B \subset H, H$ admits a bounded distortion function; polynomial or exponential distortion are defined analogously. In fact, since a change of generators results in a bilipschitz transformation of the word metric, this is then true for every choice of $A$ and $B$. Clearly the existence of a lipschitz retraction $\rho:\left(G, d_{A}\right) \rightarrow$ $\left(H, d_{B}\right)$ implies that $H$ has bounded distortion in $G$. Corollary 1.3 asserts that the converse is also true provided $H$ is word hyperbolic, i.e. the Cayley graph $\Gamma_{B}(H)$ of $H$ is $\delta$-hyperbolic for some $\delta \geq 0 . \Gamma_{B}(H)$ is a geodesic space containing an isometrically embedded copy of $\left(H, d_{B}\right)$, we may thus denote the metric on $\Gamma_{B}(H)$ by $d_{B}$ as well. The construction of the Cayley graph depends on the generating system for $H$, but the property of being word hyperbolic is, in fact, independent of the choice of $B$ (cf. [G1]). 
Proof of Corollary 1.3. We may choose the generating sets $A \subset G$ and $B \subset H$ so that $B \subset A$; then $\Gamma_{B}(H)$ may be considered as a subspace of $\Gamma_{A}(G)$. If $H$ has bounded distortion in $G$, then there exists a constant $\lambda \geq 1$ such that $d_{B}\left(x, x^{\prime}\right) \leq$ $\lambda d_{A}\left(x, x^{\prime}\right)$ for all $x, x^{\prime} \in \Gamma_{B}(H)$. Thus, if $\Gamma_{B}(H)$ is $\delta$-hyperbolic, the identity $f: \Gamma_{B}(H) \rightarrow \Gamma_{B}(H)$ possesses a $(\lambda, 3 \delta)$-lipschitz extension $\bar{f}:\left(\Gamma_{A}(G), d_{A}\right) \rightarrow$ $\left(\Gamma_{B}(H), d_{B}\right)$ by Theorem 1.2. Viewing the groups $G$ and $H$ as discrete subsets of their Cayley graphs we find a $(\lambda, 3 \delta+1)$-lipschitz retraction $\rho: G \rightarrow H$. Since $d_{A}\left(g, g^{\prime}\right) \geq 1$ for $g \neq g^{\prime}, \rho$ is a $(\lambda+3 \delta+1)$-lipschitz map.

It would be interesting to know if (or in what cases) the above retraction $\rho: G \rightarrow$ $H$ can be made $H$-equivariant, i.e. such that $\rho(h g)=h \rho(g)$ for all $g \in G$ and $h \in H$ (compare $[\mathrm{G} 2,3 . \mathrm{L}]$ ). For recent results on subgroup distortion we also refer to $[\mathrm{F}]$ and $[\mathrm{V}]$.

We conclude this section with another consequence of Theorem 1.2.

Corollary 2.3. Let $X$ be a metric space and $Y=Y_{1} \times \ldots \times Y_{n}$ the cartesian product of $n$ complete, $\delta$-hyperbolic geodesic spaces. Then every $(\lambda, \epsilon)$-lipschitz map $f: S \rightarrow Y$ defined on a subset of $X$ possesses a $(\sqrt{n} \lambda, \sqrt{n}(\epsilon+3 \delta))$-lipschitz extension $\bar{f}: X \rightarrow Y$.

Similarly, Corollary 1.3 extends to products $H=H_{1} \times \ldots \times H_{n}$ of finitely many word hyperbolic groups.

Proof. Every component $f_{i}: S \rightarrow Y_{i}$ of $f$ is $(\lambda, \epsilon)$-lipschitz and hence admits a $(\lambda, \epsilon+3 \delta)$-lipschitz extension $\bar{f}_{i}: X \rightarrow Y_{i}$ by Theorem 1.2. Then $\bar{f}:=\left(\bar{f}_{1}, \ldots, \bar{f}_{n}\right)$ : $X \rightarrow Y$ is an extension of $f$, and

$$
\begin{aligned}
d\left(\bar{f}(x), \bar{f}\left(x^{\prime}\right)\right)^{2} & =\sum_{i=1}^{n} d\left(\bar{f}_{i}(x), \bar{f}_{i}\left(x^{\prime}\right)\right)^{2} \\
& \leq \sum_{i=1}^{n}\left[\lambda d\left(x, x^{\prime}\right)+\epsilon+3 \delta\right]^{2} \\
& =n\left[\lambda d\left(x, x^{\prime}\right)+\epsilon+3 \delta\right]^{2}
\end{aligned}
$$

for $x, x^{\prime} \in X$, thus $\bar{f}$ is $(\sqrt{n} \lambda, \sqrt{n}(\epsilon+3 \delta))$-lipschitz.

In particular, if $Y$ equals the euclidean $\mathbb{R}^{n}$ (or the cartesian product of $n$ complete $\mathbb{R}$-trees), then there exists a $(\sqrt{n} \lambda, \sqrt{n} \epsilon)$-lipschitz extension $\bar{f}$ of $f$.

\section{Tight IMmersions OF GRAPHS}

Now we focus on Question 1.4. The concluding result of this paper, Theorem 4.5, provides an equivalent reformulation of the problem in the case when $X \supset S$ is a separable metric space. This criterion relies on the notion of tight immersions of finite (combinatorial) graphs which is introduced in Definition 3.1 below. Using this terminology we first formulate a necessary condition for the extendability of certain 1-lipschitz maps; cf. Proposition 3.2. Then we construct an example of a tight graph in $\mathbb{R}^{n}$ which, together with the argument proving Proposition 3.2 , yields Theorem 1.5 stated in the introduction.

For our purposes a finite graph $G$ is a pair $(V(G), E(G))$, where $V(G)$ is a nonempty finite set and $E(G)$ is a set of subsets of cardinality two of $V(G)$ whose union equals $V(G)$. The elements of $V(G)$ and $E(G)$ are called vertices and edges of $G$ respectively. A vertex $v \in V(G)$ is a terminal of $G$ if it belongs to exactly one edge, 


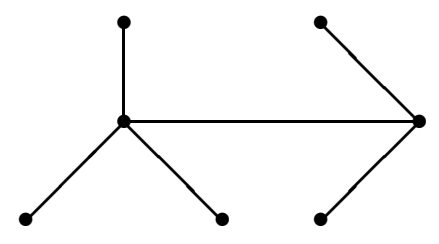

Figure 1. A graph in $\mathbb{R}^{2}$ which is partially tight but not tight.

otherwise $v$ is called an interior point of $G$ (note that, according to our definition, $G$ possesses no isolated vertices). Let $T(G)$ and $I(G)$ denote the respective subsets of $V(G)$. A path in $G$ is an ordered tuple $\left(v_{0}, v_{1}, \ldots, v_{l}\right)$ of vertices such that $\left\{v_{i-1}, v_{i}\right\} \in E(G)$ for $i=1, \ldots, l$, where $l \geq 0$. A finite graph $G$ is said to be connected if for every pair of vertices $v, w \in V(G)$ there exists a path $\left(v_{0}, \ldots, v_{l}\right)$ in $G$ from $v$ to $w$, i.e. with $v_{0}=v$ and $v_{l}=w$. A subgraph $G^{\prime}$ of $G$ is a graph with $E\left(G^{\prime}\right) \subset E(G)$; note that then $I\left(G^{\prime}\right) \subset I(G)$. Given a graph $G$, we call a map $g: V(G) \rightarrow Y$ into a set $Y$ an immersion of $G$ if $g(v) \neq g(w)$ for all $\{v, w\} \in E(G)$.

Definition 3.1. Let $G$ be a finite graph and $Y$ a metric space. We call an immersion $g: V(G) \rightarrow Y$ partially tight if there is no map $g^{\prime}: V(G) \rightarrow Y$ with $g^{\prime}(v)=g(v)$ for $v \in T(G)$ and $d\left(g^{\prime}(v), g^{\prime}(w)\right)<d(g(v), g(w))$ for all $\{v, w\} \in E(G)$. We say that $g$ is tight if there is no map $g^{\prime}: V(G) \rightarrow Y$ with $g^{\prime}(v)=g(v)$ for $v \in$ $T(G), d\left(g^{\prime}(v), g^{\prime}(w)\right) \leq d(g(v), g(w))$ for all $\{v, w\} \in E(G)$, and $d\left(g^{\prime}(v), g^{\prime}(w)\right)<$ $d(g(v), g(w))$ for at least one edge $\{v, w\} \in E(G)$.

Figure 1 shows an example of a graph $G$ embedded in $\mathbb{R}^{2}$ such that the embedding $g: V(G) \rightarrow \mathbb{R}^{2}$ is partially tight but not tight.

Note that if $g: V(G) \rightarrow Y$ is a partially tight immersion, then there is a connected component $G_{0}$ of $G$ (i.e. a maximal connected subgraph) such that $g \mid V\left(G_{0}\right)$ is still partially tight. Moreover, $g \mid T\left(G_{0}\right)$ assumes at least two distinct values; in particular, $G_{0}$ (and hence $G$ ) possesses two or more terminals. If $g$ is tight, then $g \mid V\left(G_{0}\right)$ is tight for every connected component $G_{0}$ of $G$.

Now we formulate a necessary condition for the extendability of a 1-lipschitz map $f: S \rightarrow Y$ in the case when $X \supset S$ is separable and $Y$ is a proper metric space, i.e. all closed bounded subsets are compact. (Every locally compact, complete geodesic space is proper. A proper metric space is separable.) We will show in Theorem 4.4 that the given condition is also sufficient, provided $Y$ is a convex metric space. Note that the discussion extends to $\lambda$-lipschitz maps by scaling.

Proposition 3.2. Let $Y$ be a proper metric space and $\gamma \geq 1$. Then the following assertions satisfy $(\mathrm{E}) \Leftrightarrow(\mathrm{F}) \Rightarrow(\mathrm{G})$.

(E) Every 1-lipschitz map $f: S \rightarrow Y$ defined on a subset of a separable metric space $X$ possesses a $\gamma$-lipschitz extension $\bar{f}: X \rightarrow Y$.

(F) $A s(\mathrm{E})$, but for finite $X$.

(G) If $g: V(G) \rightarrow Y$ is a partially tight immersion of a finite graph $G$, then there exists a path $\left(v_{0}, \ldots, v_{l}\right)$ in $G$ with

$$
\sum_{i=1}^{l} d\left(g\left(v_{i-1}\right), g\left(v_{i}\right)\right) \leq \gamma d\left(g\left(v_{0}\right), g\left(v_{l}\right)\right),
$$

where $v_{0}$ and $v_{l}$ are two distinct terminals of $G$. 
Proof. Since $Y$ is proper, a diagonal sequence argument shows that $(\mathrm{F}) \Rightarrow(\mathrm{E})$, and the converse is trivial.

We prove that $(\mathrm{E}) \Rightarrow(\mathrm{G})$. Let $G$ be a finite graph and $g: V(G) \rightarrow Y$ a partially tight immersion. We may assume $G$ to be connected. Since $g$ is an immersion, we can equip $V(G)$ with a metric $d^{*}$ by defining $d^{*}\left(v, v^{\prime}\right)$ to be the infimum of

$$
\sum_{i=1}^{l} d\left(g\left(v_{i-1}\right), g\left(v_{i}\right)\right)
$$

taken over all paths $\left(v_{0}, \ldots, v_{l}\right)$ in $G$ with $v_{0}=v$ and $v_{l}=v^{\prime}$. Since $G$ is finite, the infimum is always attained. Then we put

$$
c:=\min \left\{d^{*}\left(v, v^{\prime}\right) / d\left(g(v), g\left(v^{\prime}\right)\right): v, v^{\prime} \in T(G), g(v) \neq g\left(v^{\prime}\right)\right\} .
$$

In order to bring (E) into play we construct a metric space $X$ by attaching a suitably rescaled copy of $\left(V(G), d^{*}\right)$ to $Y$. To this end we first let $d^{\prime}$ denote the maximal pseudometric on $Y \cup V(G)$ satisfying $d^{\prime}\left|Y \times Y \leq d, d^{\prime}\right| V(G) \times V(G) \leq c^{-1} d^{*}$, and $d^{\prime}(g(v), v)=0$ for $v \in T(G)$. Since $d\left(g(v), g\left(v^{\prime}\right)\right) \leq c^{-1} d^{*}\left(v, v^{\prime}\right)$ for $v, v^{\prime} \in T(G)$ it follows that actually $d^{\prime} \mid Y \times Y=d$. Then we define $(X, d)$ as the metric space obtained from $\left(Y \cup V(G), d^{\prime}\right)$ by identifying points at distance zero. $X$ is a finite extension of $Y$, in particular, $X$ is separable since $Y$ is. Thus, the identity on $Y \subset X$ extends to a $\gamma$-lipschitz retraction $\rho: X \rightarrow Y$ by (E). We also have a canonical map $i: V(G) \rightarrow X$ such that

$$
d(i(v), i(w))=d^{\prime}(v, w) \leq c^{-1} d^{*}(v, w)=c^{-1} d(g(v), g(w))
$$

for $\{v, w\} \in E(G)$. The map $\rho i: V(G) \rightarrow Y$ satisfies $\rho i(v)=g(v)$ for $v \in T(G)$. Since $g$ is partially tight, there exists an edge $\{v, w\} \in E(G)$ with $d(\rho i(v), \rho i(w)) \geq$ $d(g(v), g(w))$. Using the fact that $\rho$ is $\gamma$-lipschitz we get

$$
d(g(v), g(w)) \leq d(\rho i(v), \rho i(w)) \leq \gamma d(i(v), i(w)) .
$$

By (2) and (3) we have $c \leq \gamma$. Hence, (G) holds by the definition of $c$.

Combining the final remark in section 2 with Proposition 3.2 we get

Corollary 3.3. Let $G$ be a finite graph and $g: V(G) \rightarrow \mathbb{R}^{n}$ a partially tight immersion. Then there exists a path $\left(v_{0}, \ldots, v_{l}\right)$ in $G$ with

$$
\sum_{i=1}^{l}\left|g\left(v_{i-1}\right)-g\left(v_{i}\right)\right| \leq \sqrt{n}\left|g\left(v_{0}\right)-g\left(v_{l}\right)\right|,
$$

where $v_{0}$ and $v_{l}$ are distinct terminals of $G$.

In view of Theorem 4.5, at the end of the paper it would be interesting to find a more direct (algorithmic) proof of this fact. Then one might try to generalize the argument to $n$-dimensional Hadamard manifolds; this would answer Question 1.4.

Next we describe a particular example of a tight graph in $\mathbb{R}^{n}$.

Example/Lemma 3.4. For $n \geq 2$ let $G$ be the graph with

$$
\begin{aligned}
I(G) & =\left\{x=\left(x^{1}, \ldots, x^{n}\right) \in \mathbb{R}^{n}:\left|x^{i}\right|=1 \text { for } i=1, \ldots, n\right\}, \\
T(G) & =\left\{x=\left(x^{1}, \ldots, x^{n}\right) \in \mathbb{R}^{n}:\left|x^{i}\right|=2 \text { for } i=1, \ldots, n\right\},
\end{aligned}
$$




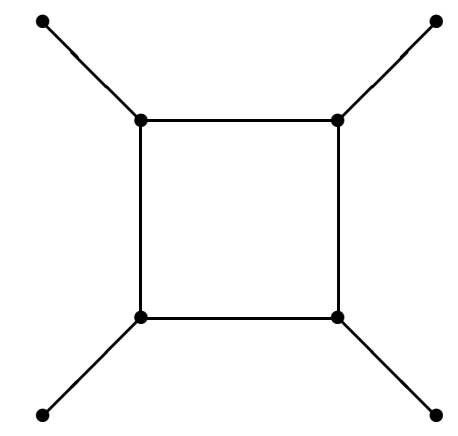

Figure 2. The graph of Example 3.4 for $n=2$.

and $\{x, y\} \in E(G)$ if and only if $x, y \in V(G)=I(G) \cup T(G)$ and either $|x-y|=2$ or $y=2 x$ (see Figure 2 for $n=2$ ). Then the inclusion $g: V(G) \rightarrow \mathbb{R}^{n}$ is tight, and whenever $\left(x_{0}, \ldots, x_{l+2}\right)$ is a path in $G$ with $x_{0}, x_{l+2} \in T(G)$ and $x_{0} \neq x_{l+2}$, then

$$
\sum_{i=0}^{l+1}\left|x_{i}-x_{i+1}\right| \geq n^{1 / 4}\left|x_{0}-x_{l+2}\right|
$$

Thus, the optimal constant measuring the distortion of the path in Corollary 3.3 lies between $n^{1 / 4}$ and $n^{1 / 2}$. The proof of 3.4 exploits the symmetry of $G$.

Proof. We show that the inclusion $g: V(G) \rightarrow \mathbb{R}^{n}$ is tight. Let $\mathcal{G}$ denote the set of maps $g^{\prime}: V(G) \rightarrow \mathbb{R}^{n}$ with $g^{\prime}(y)=y$ for $y \in T(G)$ and $\left|g^{\prime}(x)-g^{\prime}(y)\right| \leq|x-y|$ for $\{x, y\} \in E(G)$. Define $L: \mathcal{G} \rightarrow \mathbb{R}$ by

$$
L\left(g^{\prime}\right)=\sum_{\{x, y\} \in E(G)}\left|g^{\prime}(x)-g^{\prime}(y)\right| .
$$

Note that $L\left(g^{\prime}\right) \leq L(g)$ for all $g^{\prime} \in \mathcal{G}$. Clearly there exists a $g_{0} \in \mathcal{G}$ which minimizes $L$; our aim is to prove that $g_{0}=g$. First we show that $g_{0}(x) \in \mathbb{R} x$ for all $x \in I(G)$. For a fixed $x_{0} \in I(G)$ we pick an isometry $f$ of $\mathbb{R}^{n}$ such that $f(V(G))=V(G)$, $f\left(r x_{0}\right)=r x_{0}$ for $r \in \mathbb{R}$, and $f(y) \neq y$ for $y \notin \mathbb{R} x_{0}$. It is readily checked that both $f g_{0} f^{-1} \mid V(G)$ and

$$
g_{0}^{\prime}:=\left[g_{0}+f g_{0} f^{-1} \mid V(G)\right] / 2
$$

are elements of $\mathcal{G}$. Now suppose that $g_{0}\left(x_{0}\right) \notin \mathbb{R} x_{0}$. Then $f g_{0} f^{-1}\left(x_{0}\right)=f g_{0}\left(x_{0}\right) \neq$ $g_{0}\left(x_{0}\right)$ by the choice of $f$. Since $\left|f g_{0} f^{-1}\left(x_{0}\right)-2 x_{0}\right|=\left|g_{0}\left(x_{0}\right)-2 x_{0}\right|$ it follows that $\left|g_{0}^{\prime}\left(x_{0}\right)-2 x_{0}\right|<\left|g_{0}\left(x_{0}\right)-2 x_{0}\right|$. Using this inequality one shows that $L\left(g_{0}^{\prime}\right)<L\left(g_{0}\right)$, in contradiction to the choice of $g_{0}$. Therefore $g_{0}(x) \in \mathbb{R} x$ for all $x \in I(G)$. In fact, since $\left|g_{0}(x)-2 x\right| \leq|x-2 x|=|x|$, we see that $g_{0}(x) \in[x, 3 x]$ for all $x \in I(G)$. Now $g_{0}\left(x_{0}\right) \neq x_{0}$ for some $x_{0} \in I(G)$ would imply $\left|g_{0}\left(x_{0}\right)-g_{0}(y)\right|>\left|x_{0}-y\right|$ for all $y \in I(G)$ with $\left\{x_{0}, y\right\} \in E(G)$, hence $g_{0}=g$. In particular, if $g^{\prime} \in \mathcal{G}$, then $L(g) \geq L\left(g^{\prime}\right) \geq L\left(g_{0}\right)=L(g)$ and thus $\left|g^{\prime}(x)-g^{\prime}(y)\right|=|x-y|$ for all $\{x, y\} \in E(G)$. This shows that $g$ is tight, in fact it follows that $\mathcal{G}=\{g\}$.

To prove the claimed inequality it suffices to consider paths $\left(x_{0}, \ldots, x_{l+2}\right)$ with $x_{1}, \ldots, x_{l+1} \in I(G)$. Then clearly

$$
\sum_{i=0}^{l+1}\left|x_{i}-x_{i+1}\right|=\left|x_{0}-x_{1}\right|+\left|x_{l+1}-x_{l+2}\right|+\sum_{i=1}^{l}\left|x_{i}-x_{i+1}\right|=2(\sqrt{n}+l)
$$


and $\left|x_{0}-x_{l+2}\right| \leq 4 \sqrt{l}$. One checks that $q_{n}(l):=(\sqrt{n}+l) /(2 \sqrt{l}) \geq q_{n}(\sqrt{n})=n^{1 / 4}$ for all $l>0$, which yields the claim.

In order to prove Theorem 1.5 stated in the introduction, we use the procedure described in the proof of Proposition 3.2 to attach larger and larger copies of the above examples to $\mathbb{R}^{n}$ and $\mathbb{R}^{\infty}$ respectively. In the latter case the dimension is increased as well. We leave the details to the reader.

\section{MAPs into CONVEX SPACES}

The main purpose of this last section is to prove the "missing" implication in Proposition 3.2; to this end we assume $Y$ to be a convex metric space. This means that $Y$ is geodesic and for every pair of (constant speed) geodesics $\sigma, \sigma^{\prime}:[0,1] \rightarrow Y$,

$$
d\left(\sigma(t), \sigma^{\prime}(t)\right) \leq(1-t) d\left(\sigma(0), \sigma^{\prime}(0)\right)+t d\left(\sigma(1), \sigma^{\prime}(1)\right)
$$

for all $t \in[0,1]$. In particular, it follows that for every pair of points $x, y \in Y$ there is actually a unique geodesic $\sigma:[0,1] \rightarrow Y$ from $x$ to $y$. The class of convex metric spaces was first studied by Busemann $[\mathrm{Bu}]$ and includes Hadamard manifolds, or Hadamard spaces in the sense of Alexandrov, and Banach spaces with a strictly convex norm.

The following fact is well-known, cf. $[\mathrm{Bu},(36.6)]$ or $[\mathrm{J}]$.

Lemma 4.1. Let $Y$ be a convex metric space and $\sigma_{1}, \sigma_{2}:[0,1] \rightarrow Y$ two geodesics with $\sigma_{1}(0)=\sigma_{2}(0)=: z$, $\operatorname{im} \sigma_{1} \not \subset \operatorname{im} \sigma_{2}$ and $\operatorname{im} \sigma_{2} \not \subset \operatorname{im} \sigma_{1}$. Let $m$ be the midpoint between $\sigma_{1}(1)$ and $\sigma_{2}(1)$. Then $d(m, z)<\left[d\left(\sigma_{1}(1), z\right)+d\left(\sigma_{2}(1), z\right)\right] / 2$.

Proof. Since $\operatorname{im} \sigma_{1} \not \subset \operatorname{im} \sigma_{2}$ and $\operatorname{im} \sigma_{2} \not \subset \operatorname{im} \sigma_{1}$ there exist $t_{1}, t_{2} \in[0,1)$ such that $\sigma_{1}\left(t_{1}\right)=\sigma_{2}\left(t_{2}\right)=: y$ and $\sigma_{1}\left(\left(t_{1}, 1\right]\right) \cap \sigma_{2}\left(\left(t_{2}, 1\right]\right)=\emptyset$. Then at least one of the points $y_{1}:=\sigma_{1}\left(\left(t_{1}+1\right) / 2\right)$ and $y_{2}:=\sigma_{2}\left(\left(t_{2}+1\right) / 2\right)$ does not lie on the segment $[y, m]$, hence

$$
2 d(y, m)<d\left(y, y_{1}\right)+d\left(y_{1}, m\right)+d\left(y, y_{2}\right)+d\left(y_{2}, m\right) .
$$

By convexity, $d\left(y_{1}, m\right) \leq d\left(y, \sigma_{2}(1)\right) / 2$ and $d\left(y_{2}, m\right) \leq d\left(y, \sigma_{1}(1)\right) / 2$. It follows that

$$
2 d(y, m)<d\left(y, \sigma_{1}(1)\right)+d\left(y, \sigma_{2}(1)\right)
$$

which implies the claim.

The next two results provide some additional information about (partially) tight immersions of a finite graph into a convex metric space; they are included for illustration and will not be used in the sequel.

Lemma 4.2. Let $G$ be a finite graph and $g: V(G) \rightarrow Y$ an immersion into a convex metric space $Y$. Then $g$ is tight if and only if the following holds: whenever $g^{\prime}: V(G) \rightarrow Y$ is a map with $g^{\prime}(v)=g(v)$ for $v \in T(G)$ and $d\left(g^{\prime}(v), g^{\prime}(w)\right) \leq$ $d(g(v), g(w))$ for $\{v, w\} \in E(G)$, then $g^{\prime}=g$.

Proof. If the given condition holds, then $g$ is obviously tight. Conversely, if $g$ is tight, and if $g^{\prime}: V(G) \rightarrow Y$ is a map with $g^{\prime}(v)=g(v)$ for $v \in T(G)$ and $d\left(g^{\prime}(v), g^{\prime}(w)\right) \leq d(g(v), g(w))$ for $\{v, w\} \in E(G)$, then actually $d\left(g^{\prime}(v), g^{\prime}(w)\right)=$ $d(g(v), g(w))$ for all $\{v, w\} \in E(G)$. Suppose that $g^{\prime}\left(v_{0}\right) \neq g\left(v_{0}\right)$ for some $v_{0} \in I(G)$. Since $g$ is tight, the connected component $G_{0}$ of $G$ containing $v_{0}$ possesses a terminal $\bar{v}$. Picking a path $\left(v_{0}, \ldots, v_{l}\right)$ with $v_{l}=\bar{v}$ we find an index $i$ such that $g^{\prime}\left(v_{i}\right) \neq g\left(v_{i}\right)$ and $g^{\prime}\left(v_{i+1}\right)=g\left(v_{i+1}\right)$. Using the convexity of $Y$ and Lemma 4.1 we see that the map $m: V(G) \rightarrow Y$ averaging $g$ and $g^{\prime}$ satisfies $d(m(v), m(w)) \leq d(g(v), g(w))$ for 
all $\{v, w\} \in E(G)$ and $d\left(m\left(v_{i}\right), m\left(v_{i+1}\right)\right)<d\left(g\left(v_{i}\right), g\left(v_{i+1}\right)\right)$, which is impossible since $g$ is tight. Therefore $g^{\prime}=g$.

Lemma 4.3. Let $G$ be a finite graph and $g: V(G) \rightarrow Y$ a partially tight immersion into a convex metric space $Y$. Then $G$ contains a connected subgraph $G_{0}$ such that $T\left(G_{0}\right) \subset T(G)$ and $g \mid V\left(G_{0}\right)$ is tight.

Proof. Let $F \subset E(G)$ be a maximal set (not contained in a bigger one) with the property that there exists a map $g^{\prime}: V(G) \rightarrow Y$ such that $g^{\prime}(v)=g(v)$ for $v \in T(G), d\left(g^{\prime}(v), g^{\prime}(w)\right) \leq d(g(v), g(w))$ for $\{v, w\} \in E(G)$, and $d\left(g^{\prime}(v), g^{\prime}(w)\right)<$ $d(g(v), g(w))$ for $\{v, w\} \in F$. Since $g$ is partially tight, $F \neq E(G)$. Consider the graph $G^{\prime}$ with edge set $E\left(G^{\prime}\right)=E(G) \backslash F$; it follows easily from the finiteness of $G$ and the maximality of $F$ that $T\left(G^{\prime}\right) \subset T(G)$. We show that $g \mid V\left(G^{\prime}\right)$ is tight. Suppose this is not the case. Then there exists a map $g^{\prime \prime}: V\left(G^{\prime}\right) \rightarrow Y$ with $g^{\prime \prime}(v)=g(v)$ for $v \in T\left(G^{\prime}\right), d\left(g^{\prime \prime}(v), g^{\prime \prime}(w)\right) \leq d(g(v), g(w))$ for $\{v, w\} \in E\left(G^{\prime}\right)$, and $d\left(g^{\prime \prime}\left(v^{\prime}\right), g^{\prime \prime}\left(w^{\prime}\right)\right)<d\left(g\left(v^{\prime}\right), g\left(w^{\prime}\right)\right)$ for some $\left\{v^{\prime}, w^{\prime}\right\} \in E\left(G^{\prime}\right)$. By the convexity of $Y$ we may assume that $d\left(g^{\prime \prime}(v), g(v)\right)<\epsilon / 2$ for all $v \in V\left(G^{\prime}\right)$, where $\epsilon$ is chosen such that

$$
d\left(g^{\prime}(v), g^{\prime}(w)\right) \leq d(g(v), g(w))-\epsilon
$$

for all $\{v, w\} \in F$. Then we extend $g^{\prime \prime}$ to all of $V(G)$ by defining $g^{\prime \prime}(v)=g(v)$ for $v \in V(G) \backslash V\left(G^{\prime}\right)$. Note that

$$
d\left(g^{\prime \prime}(v), g^{\prime \prime}(w)\right)<d(g(v), g(w))+\epsilon
$$

for all $\{v, w\} \in E(G)$. The map $m: V(G) \rightarrow Y$ sending $v$ to the midpoint between $g^{\prime}(v)$ and $g^{\prime \prime}(v)$ satisfies

$$
d(m(v), m(w)) \leq\left[d\left(g^{\prime}(v), g^{\prime}(w)\right)+d\left(g^{\prime \prime}(v), g^{\prime \prime}(w)\right)\right] / 2
$$

for all $\{v, w\} \in E(G)$. It follows that $d(m(v), m(w)) \leq d(g(v), g(w))$ for $\{v, w\} \in$ $E(G)$ and $d(m(v), m(w))<d(g(v), g(w))$ for $\{v, w\} \in F$ or $\{v, w\}=\left\{v^{\prime}, w^{\prime}\right\}$. Since $m(v)=g(v)$ for $v \in T(G)$, this contradicts the choice of $F$. Therefore $g \mid V\left(G^{\prime}\right)$ is tight. Finally, we pick an arbitrary connected component $G_{0}$ of $G^{\prime}$. Then $g \mid V\left(G_{0}\right)$ is still tight, and $T\left(G_{0}\right) \subset T\left(G^{\prime}\right) \subset T(G)$.

Now we prove

Theorem 4.4. Let $Y$ be a proper, convex metric space and $\gamma \geq 1$. Then the assertions $(\mathrm{E}),(\mathrm{F})$, and $(\mathrm{G})$ of Proposition 3.2 are equivalent.

Proof. It remains to show that (G) implies (F). Let $f: S \rightarrow Y$ be a 1-lipschitz map defined on a subset $S$ of a finite metric space $X$. We denote the elements of $X \backslash S$ by $x_{1}, \ldots, x_{m}$. Let $\beta \geq 0$. We define

$$
A_{\beta}:=\left\{\left(y_{1}, \ldots, y_{m}\right) \in Y^{m}: d\left(y_{i}, y_{j}\right) \leq \beta d\left(x_{i}, x_{j}\right) \text { for } 1 \leq i<j \leq m\right\},
$$

and for $z \in S$ we put

$$
B_{\beta}(z):=\left\{\left(y_{1}, \ldots, y_{m}\right) \in Y^{m}: d\left(y_{i}, f(z)\right) \leq \beta d\left(x_{i}, z\right) \text { for } 1 \leq i \leq m\right\} .
$$

Finally, we let

$$
C_{\beta}:=A_{\beta} \cap \bigcap_{z \in S} B_{\beta}(z) .
$$

We claim that, due to assertion $(\mathrm{G}), C_{\gamma} \neq \emptyset$. If this is true, then we may pick a point $\bar{y}=\left(y_{1}, \ldots, y_{m}\right) \in C_{\gamma}$ and extend $f$ to $X$ by defining $\bar{f}\left(x_{i}\right):=y_{i}$ for 
$i=1, \ldots, m$; then clearly $\bar{f}$ is $\gamma$-lipschitz and (F) holds. It is easily seen that $C_{\beta}$ is non-empty for $\beta$ sufficiently large. Let

$$
\gamma^{\prime}:=\inf \left\{\beta \geq 0: C_{\beta} \neq \emptyset\right\} .
$$

Since $Y$ is proper the sets $C_{\beta}$ are compact, moreover $C_{\beta^{\prime}} \subset C_{\beta}$ for $\beta^{\prime} \leq \beta$, and $C_{\gamma^{\prime}}=\bigcap_{i=1}^{\infty} C_{\gamma^{\prime}+1 / i}$. From this we see that $C_{\gamma^{\prime}} \neq \emptyset$; it remains to show that $\gamma^{\prime} \leq \gamma$.

We suppose that $\gamma^{\prime}>0$ and choose a point $\bar{y}=\left(y_{1}, \ldots, y_{m}\right) \in C_{\gamma^{\prime}}$. Denoting the elements of $S$ by $z_{1}, \ldots, z_{n}$ we construct a finite graph $G$ with vertex set

$$
V(G) \subset \bar{V}:=\left\{v_{i k}: i=1, \ldots, m, k=0, \ldots, n\right\}
$$

and edge set $E(G)$ given as follows:

(a) if $1 \leq i<j \leq m$ and $d\left(y_{i}, y_{j}\right)=\gamma^{\prime} d\left(x_{i}, x_{j}\right)$, then $\left\{v_{i 0}, v_{j 0}\right\} \in E(G)$,

(b) if $1 \leq i \leq m, 1 \leq k \leq n$, and $d\left(y_{i}, f\left(z_{k}\right)\right)=\gamma^{\prime} d\left(x_{i}, z_{k}\right)$, then $\left\{v_{i 0}, v_{i k}\right\} \in$ $E(G)$

and this determines already all elements of $E(G)$. Then we define $g: V(G) \rightarrow Y$ by $g\left(v_{i 0}\right)=y_{i}$ and $g\left(v_{i k}\right)=f\left(z_{k}\right)$ for $k \geq 1$. Note that $g$ is an immersion of $G$ since $\gamma^{\prime}>0$. Assume that one of the vertices $v_{i 0} \in V(G)$ belongs to exactly one edge $\left\{v_{i 0}, w\right\} \in E(G)$. Then we can choose a point $y_{i}^{\prime} \neq y_{i}$ on the geodesic segment $\left[y_{i}, g(w)\right]$ such that $\bar{y}^{\prime}:=\left(y_{1}, \ldots, y_{i-1}, y_{i}^{\prime}, y_{i+1}, \ldots, y_{m}\right)$ still belongs to the set $C_{\gamma^{\prime}}$, and, if we repeat the above construction of $G$ with $\bar{y}$ replaced by $\bar{y}^{\prime}$, the resulting graph $G^{\prime}$ is a subgraph of $G$ with $v_{i 0} \notin V\left(G^{\prime}\right)$. This shows that we may assume every vertex $v_{i 0} \in V(G)$ to be an interior point of $G$. On the other hand, the vertices $v_{i k} \in V(G)$ with $k \geq 1$ are terminals of $G$.

We claim that $g$ is partially tight. If not, then there exists a map $g^{\prime}: V(G) \rightarrow Y$ with $g^{\prime}(v)=g(v)$ for $v \in T(G)$ and $d\left(g^{\prime}(v), g^{\prime}(w)\right)<d(g(v), g(w))$ for $\{v, w\} \in$ $E(G)$. Since $Y$ is a convex space we may assume that $d\left(g^{\prime}(v), g(v)\right)<\alpha$ for all $v \in V(G)$, where we choose $\alpha$ such that the following holds:

(a) if $1 \leq i<j \leq m$ and $\left\{v_{i 0}, v_{j 0}\right\} \notin E(G)$, then $d\left(y_{i}, y_{j}\right) \leq \gamma^{\prime} d\left(x_{i}, x_{j}\right)-\alpha$,

(b) if $1 \leq i \leq m, 1 \leq k \leq n$, and $\left\{v_{i 0}, v_{i k}\right\} \notin E(G)$, then $d\left(y_{i}, f\left(z_{k}\right)\right) \leq$ $\gamma^{\prime} d\left(x_{i}, z_{k}\right)-\alpha$.

We extend $g^{\prime}$ to all of $\bar{V}$ by defining $g^{\prime}\left(v_{i 0}\right)=y_{i}$ for $v_{i 0} \notin V(G)$ and $g^{\prime}\left(v_{i k}\right)=f\left(z_{k}\right)$ for $v_{i k} \notin V(G)$ and $k \geq 1$. It is not difficult to check that then $\left(g^{\prime}\left(v_{10}\right), \ldots, g^{\prime}\left(v_{m 0}\right)\right)$ belongs to $C_{\beta}$ for some $\beta<\gamma^{\prime}$, which contradicts the definition of $\gamma^{\prime}$. Therefore $g$ is a partially tight immersion.

According to assertion $(\mathrm{G})$, there exists a path $\left(v_{0}, \ldots, v_{l}\right)$ in $G$ with

$$
\sum_{i=1}^{l} d\left(g\left(v_{i-1}\right), g\left(v_{i}\right)\right) \leq \gamma d\left(g\left(v_{0}\right), g\left(v_{l}\right)\right),
$$

where $v_{0}$ and $v_{l}$ are distinct terminals of $G$. Let $h: V(G) \rightarrow X$ be defined by $h\left(v_{i 0}\right)=x_{i}$ and $h\left(v_{i k}\right)=z_{k}$ for $k \geq 1$. Since the vertices $v_{i 0} \in V(G)$ are interior points of $G$, we have $v_{0}=v_{i(0) k(0)}$ and $v_{l}=v_{i(l) k(l)}$ for some $k(0), k(l) \geq 1$. Therefore $f h\left(v_{0}\right)=f\left(z_{k(0)}\right)=g\left(v_{0}\right)$ and $f h\left(v_{l}\right)=g\left(v_{l}\right)$. Hence, using the fact that $f$ is 1-lipschitz and the triangle inequality for $X$, we get

$$
d\left(g\left(v_{0}\right), g\left(v_{l}\right)\right) \leq d\left(h\left(v_{0}\right), h\left(v_{l}\right)\right) \leq \sum_{i=1}^{l} d\left(h\left(v_{i-1}\right), h\left(v_{i}\right)\right) .
$$

On the other hand, by the definition of the graph $G$, we have

$$
d\left(g\left(v_{i-1}\right), g\left(v_{i}\right)\right)=\gamma^{\prime} d\left(h\left(v_{i-1}\right), h\left(v_{i}\right)\right)
$$


for $i=1, \ldots, l$. Combining (4), (5), and (6) we see that $\gamma^{\prime} \leq \gamma$.

We conclude this paper with an equivalent reformulation of Question 1.4 in terms of assertion $(\mathrm{G})$. A family $\mathcal{Y}$ of metric spaces is called scale invariant if $(Y, d) \in \mathcal{Y}$ implies that $(Y, c \cdot d) \in \mathcal{Y}$ for all $c>0$.

Theorem 4.5. Let $\mathcal{Y}$ be a scale invariant family of proper, convex metric spaces. Then the following are equivalent:

(i) For all $\lambda, \epsilon$ there exist $\bar{\lambda}, \bar{\epsilon}$ such that if $X$ is a separable metric space and $Y \in \mathcal{Y}$, then every $(\lambda, \epsilon)$-lipschitz map $f: S \rightarrow Y$ with $S \subset X$ possesses a $(\bar{\lambda}, \bar{\epsilon})$-lipschitz extension $\bar{f}: X \rightarrow Y$.

(ii) There exists a constant $\mu \geq 1$ such that (i) holds for $\bar{\lambda}=\mu \lambda$ and $\bar{\epsilon}=\mu \epsilon$.

(iii) There exists a constant $\gamma \geq 1$ such that assertion (G) of Proposition 3.2 holds for all $Y \in \mathcal{Y}$.

This applies, for instance, if $\mathcal{Y}$ is the class of all Hadamard manifolds of a fixed dimension $n$.

Proof. From Theorem 4.4 we know that (iii) is equivalent to the following:

(iv) There exists a constant $\gamma \geq 1$ such that if $X$ is a finite metric space and $Y \in \mathcal{Y}$, then every 1-lipschitz map $f: S \rightarrow Y$ with $S \subset X$ possesses a $\gamma$ lipschitz extension $\bar{f}: X \rightarrow Y$.

The implication (ii) $\Rightarrow$ (i) is trivial; we prove that (i) $\Rightarrow$ (iv). If $f$ is given as in (iv), then by (i) there exists a $(\gamma, \delta)$-lipschitz extension $\bar{f}: X \rightarrow Y$ of $f$, where $\gamma=\bar{\lambda}(1,0)$ and $\delta=\bar{\epsilon}(1,0)$. In fact, by multiplying the metric on both $X$ and $Y$ by a factor $c>1$, applying this result, and scaling back, we can replace $\delta$ by $\delta / c$. Since $X$ is finite and $Y$ proper, we can as well achieve that $\delta=0$.

It remains to show that (iv) $\Rightarrow$ (ii). First we note that, due to (iv), every $(\lambda, \epsilon)$ lipschitz map $f: S \rightarrow Y$ defined on a subset of a finite metric space $X$ can be extended to a $(\gamma \lambda, \gamma \epsilon)$-lipschitz map $\bar{f}: X \rightarrow Y$. This follows easily by looking at the metric $d^{\prime}$ on $X$ satisfying $d^{\prime}\left(x, x^{\prime}\right)=\lambda d\left(x, x^{\prime}\right)+\epsilon$ for $x \neq x^{\prime}$. Now let $f$ be given as in (ii). Since $X$ is separable and $Y$ is proper, a diagonal sequence argument produces a $(\gamma \lambda, \gamma \epsilon)$-lipschitz map $f^{\prime}: C \rightarrow Y$, where $C$ is a countable dense subset of $X, C \cap S$ is dense in $S$, and $f^{\prime}|(C \cap S)=f|(C \cap S)$. Thus, if $\epsilon=0$, then $f^{\prime}$ possesses a unique $\gamma \lambda$-lipschitz extension $\bar{f}: X \rightarrow Y$ which is also an extension of $f$. If $\epsilon>0$, we pick an $\alpha>0$ together with a map $h: X \rightarrow C$ satisfying $d(h(x), x) \leq \alpha$ for all $x$ and $h(z) \in C \cap S$ for $z \in S$. Then we define $\bar{f}: X \rightarrow Y$ so that $\bar{f}\left|(X \backslash S)=f^{\prime} h\right|(X \backslash S)$ and $\bar{f} \mid S=f$. If $x, x^{\prime} \in X \backslash S$, then

$$
\begin{aligned}
d\left(\bar{f}(x), \bar{f}\left(x^{\prime}\right)\right) & =d\left(f^{\prime} h(x), f^{\prime} h\left(x^{\prime}\right)\right) \\
& \leq \gamma \lambda d\left(h(x), h\left(x^{\prime}\right)\right)+\gamma \epsilon \\
& \leq \gamma \lambda d\left(x, x^{\prime}\right)+2 \gamma \lambda \alpha+\gamma \epsilon .
\end{aligned}
$$

For $x \in X \backslash S$ and $z \in S$,

$$
\begin{aligned}
d(\bar{f}(x), \bar{f}(z)) & \leq d\left(f^{\prime} h(x), f^{\prime} h(z)\right)+d(f h(z), f(z)) \\
& \leq \gamma \lambda d(h(x), h(z))+\gamma \epsilon+\lambda d(h(z), z)+\epsilon \\
& \leq \gamma \lambda d(x, z)+2 \gamma \lambda \alpha+\gamma \epsilon+\lambda \alpha+\epsilon .
\end{aligned}
$$

Hence, choosing $\mu>\gamma+1$, we conclude that $\bar{f}$ is a $(\mu \lambda, \mu \epsilon)$-lipschitz extension of $f$ for $\alpha$ sufficiently small. 


\section{REFERENCES}

[B] B. Bowditch: Notes on Gromov's hyperbolicity criterion, in: E. Ghys, A. Haefliger, A. Verjovsky (eds.), Group theory from a geometrical viewpoint, Singapore: World Scientific 1991, pp. 64-167. MR 93h:57002

[Bu] H. Busemann: The geometry of geodesics, New York: Academic Press 1955. MR 17:779a

[F] B. Farb: The extrinsic geometry of subgroups and the generalized word problem, Proc. London Math. Soc. 68 (1994), 577-593. MR 94m:20073

[G1] M. Gromov: Hyperbolic groups, in: S. M. Gersten (ed.), Essays in group theory, MSRI Publ. no. 8, New York: Springer 1987, pp. 75-263. MR 89e:20070

[G2] : Asymptotic invariants of infinite groups, in: G. A. Niblo, M. A. Roller (eds.), Geometric group theory, vol. 2, London Math. Soc. Lecture Note Series no. 182, Cambridge Univ. Press 1993, pp. 1-295. MR 95m:20041

[J] J. Jost: Nonpositive curvature. Geometric and analytic aspects, Lectures in Math., ETH Zürich, Basel: Birkhäuser 1997. MR 98g:53070

[K] M. D. Kirszbraun: Über die zusammenziehende und Lipschitzsche Transformationen, Fundamenta Math. 22 (1934), 77-108.

[LS] U. Lang, V. Schroeder: Kirszbraun's theorem and metric spaces of bounded curvature, Geom. Funct. Anal. (GAFA) 7 (1997), 535-560. MR 98d:53062

[M] E. J. McShane: Extension of range of functions, Bull. Amer. Math. Soc. 40 (1934), 837842.

[MoSh] J. W. Morgan, P. B. Shalen: Valuations, trees, and degenerations of hyperbolic structures, I, Ann. of Math. 120 (1984), 401-476. MR 86f:57011

[V] N. Varopoulos: Sur la distortion de distances des sous-groupes des groupes de Lie, C. R. Acad. Sci. Paris Sér. I Math. 322 (1996), 1025-1026. MR 97d:22010

Departement Mathematik, Eidgen Technische Hochschule Zentrum, CH-8092 Zürich, SWITZERLAND

E-mail address: lang@math.ethz.ch 